\title{
Hypoxia, hibernation and Neuroprotection: An Experimental Study in Mice
}

\author{
Changhong Ren ${ }^{1,2}$, Sijie Li ${ }^{1}$, Gary Rajah ${ }^{3}$, Guo Shao ${ }^{1}$, Guowei Lu', Rongrong Han', Qingjian \\ Huang ${ }^{1}$, Haiyan $\mathrm{Li}^{1}$, Yuchuan Ding ${ }^{1,3}$, Kunlin Jin ${ }^{1,4}$, Xunming Ji ${ }^{1,2, *}$ \\ ${ }^{1}$ Beijing Key Laboratory of Hypoxia Translational Medicine, Beijing 100053, China \\ ${ }^{2}$ Center of Stroke, Beijing Institute for Brain Disorder, Beijing 100069, China \\ ${ }^{3}$ Department of Neurosurgery, Wayne State University School of Medicine, Detroit, MI 48201, USA \\ ${ }^{4}$ Department of Pharmacology and Neuroscience, University of North Texas Health Science Center, Fort Worth, \\ TX 76107, USA
}

[Received April 12, 2018; Revised July 1, 2018; Accepted July 2, 2018]

\begin{abstract}
Hibernation is a unique physiological state that evolved to survive periods of food shortages. It is characterized by profound decreases in metabolic rate, body temperature and physiological functions. Studies have shown that animals in hibernation can resist neurological damage. Here, we aimed to study whether hypoxia can induce a hibernation-like state in a traditionally non-hibernating animal and whether it is neuroprotective. All procedures were conducted according to international guidelines on laboratory animal safety. Mice C57BL/6 (19-21g) were placed into a $125 \mathrm{~mL}$ jar with fresh air and the jar was sealed with a rubber plug. For each run, the tolerance limit was judged by the animals' appearance for "air hunger". The animal was removed from the jar as soon as its first gasping breath appeared and was moved to another fresh-air-containing jar of similar volume. This procedure was performed in four runs. The hypoxia exposure significantly decreased oxygen $\left(\mathrm{O}_{2}\right)$ consumption, carbon dioxide $\left(\mathrm{CO}_{2}\right)$ production, respiratory rate and heart rate. Meanwhile, rectal temperature reached a minimum of $12.7 \pm 2.56^{\circ} \mathrm{C}$, which is lower than a wide range of ambient temperatures. The mimicked hibernation decreased the infarct size in a focal cerebral ischemia mouse model. Our findings suggest the possibility of inducing suspended animation-like hibernation states for medical applications post injury.
\end{abstract}

Key words: hibernation, hypothermia, hypoxia, clinical application

Endothermic mammals have the ability to maintain a constant high body temperature $\left(\mathrm{T}_{\mathrm{b}}\right)$ over a wide range of ambient temperatures $\left(\mathrm{T}_{\mathrm{a}}\right)$ [1]. However, in the cold winter or food-deficient season, some mammals no longer maintain permanent homeothermy and enter a state of hibernation. Hibernation in these heterothermic endothermic animals is characterized by a controlled reduction of $\mathrm{T}_{\mathrm{b}}$ via the metabolic rate $[2,3]$. In the winter, the core body temperature of bears reduce to $\sim 32^{\circ} \mathrm{C}$, and the tropical Malagasy lemur, a primate that hibernates in the dry season of the tropics, drops its core body temperature to around $25^{\circ} \mathrm{C}$ [4]. Arctic ground squirrels can withstand drops in core body temperature to several degrees below freezing point [3]. The above mammals can enter severe hypothermic states during hibernation in which metabolic activities are extremely low, and yet full viability is restored when the animals arouse from such a state [5]. Numerous studies have shown that the hibernation state has great medical benefits for a variety of conditions including several neuroprotective adaptations (i.e. ischemia/reperfusion, trauma, neurodegenerative diseases) [6-9]. Furthermore, hibernation is a promising area for neuroprotection in traumatic brain injury and stroke as suggested by the National Institutes of Health [7]. The hibernation-like states may also be useful in surgical situations and for

*Correspondence should be addressed to: Dr. Xunming Ji, Institute of Hypoxia Medicine, Xuanwu Hospital, Capital Medical University, 45 Changchun Road, Xicheng District, Beijing 100053, China. E-mail: jixm@ccmu.edu.cn

Copyright: ( 2018 Ren C et al. This is an open-access article distributed under the terms of the Creative Commons Attribution License, which permits unrestricted use, distribution, and reproduction in any medium, provided the original author and source are credited. 
improving organ preservation for transplantation [10]. Therefore, if non-hibernators can be induced into a hibernation state with similar benefits on metabolism and post hibernation function as true hibernators, this therapy could prove promising for many ailments.

In the 1960s, Dawe et al first induced a hibernation state in a non-hibernator, through injection of serum from torpid animals [11]. From then on, researchers were exploring several methods to induce a hibernation-like state in non-hibernators. How hibernation occurs in nonhibernating animals remains unknown. Hypoxia exposure has been studied for years including detrimental and beneficial effects [12]. Non-lethal hypoxia is well known to lead a drop in $\mathrm{T}_{\mathrm{b}}$ in newborns and adults of many species including humans $[13,14]$. This hypoxia-induced drop in $\mathrm{T}_{\mathrm{b}}$ and metabolic depression serves a protective role by reducing $\mathrm{O}_{2}$ demand, eliminating costly thermogenesis, improving blood $\mathrm{O}_{2}$ affinity, and reducing the costs of ventilation $[15,16]$. Tattersall et al reported that hypoxia exposure $\left(120 \mathrm{~min}, 7 \% \mathrm{O}_{2}\right)$ caused body temperature to drop from the normoxic value of 37.7 to $31.9^{\circ} \mathrm{C}$. This was accompanied by a marked metabolic depression $\left(\mathrm{O}_{2}\right.$ consumption was $46 \%$ of the normoxic value). These data suggested that controlled hypoxia exposure is a potential method for inducing a hibernationlike state. However, there is no current evidence that a lack of oxygen is a suitable stimulus for entrance into a hibernation-like physiological state [4].

Our study utilizes the development of a unique animal model of hypoxic exposure to assess if nonhibernators could in fact be made to enter a hibernationlike state following controlled hypoxia. We also explored the potential application of the hibernation-like state.

\section{MATERIALS AND METHODS}

\section{Animal model}

All animal experiments were approved by the Institutional Animal Care and Use Committee of Xuanwu Hospital, Capital Medical University, China, and conducted according to guidelines laid out by the National Institutes of Health. Male mice C57BL/6 (19-21g) were used in this study (Vital River Laboratories, Beijing, China). Animals were maintained on a 12-hour light/dark cycle with unlimited access to food and water.

The procedure for hypoxic exposure was performed as previously described [17]. Briefly, the male mice were placed into a $125 \mathrm{~mL}$ jar with fresh air, and the jar was sealed with a rubber plug. The tolerance limit was judged by the animals' appearance of ' gasping breath or air hunger" for each run. The animal was removed from the jar as soon as its first gasping breath appeared and was moved to another fresh-air-containing jar of similar volume. This procedure was performed once (H1) and repeated two, three and four times ( $\mathrm{H} 2$ or $\mathrm{H} 3$ and $\mathrm{H} 4)$, respectively. A blank control group with no exposure to hypoxia (H0) was put into the jar without a lid on. The time between the beginning of airtightness and the appearance of the first gasping was termed "tolerance time" for each run. Ambient temperature was maintained at $20 \pm 0.5^{\circ} \mathrm{C}$ and concentration of $\mathrm{O}_{2}$ was $21 \%$ for room air.

\section{Core body temperature measurement}

The rectal temperature of a mouse was measured with a measurement probe immediately after each run (Harvard Apparatus, Holliston, MA, USA).

\section{Physiological index detection}

Blood pressure was measured immediately after removal from the jar using a standard tail cuff (BP-2010A System, Softron, Beijing, CHINA). Heart rate, respiratory rate, $\mathrm{O}_{2}$ consumption and $\mathrm{CO}_{2}$ production were measured as previously described [18].

\section{Distal middle cerebral artery occlusion in the mouse}

For distal middle cerebral artery occlusion (dMCAO), C57BL/6 (20-22 g) mice were used in this study as described previously [19]. The mice underwent hypoxia exposure for 4 runs at 30 min per run after dMCAO surgery.

\section{Infarct size measurement}

Infarct size was measured with a $2 \%$ solution of $2,3,4-$ triphenytetrazolium-chloride at $24 \mathrm{~h}$ after dMCAO as described previously [20].

\section{Adhesive tape removal test}

Sensorimotor functional recovery after stroke was measured at 3 days before $\mathrm{dMCAO}$ and at 3 days after dMCAO with the adhesive tape removal test as previously described $[21,22]$.

\section{Statistical analysis}

Data was expressed as mean \pm standard deviation (mean \pm SD) and statistical tests were performed with SPSS for Windows, version 17.0 (SPSS Inc.). One-way ANOVA followed by the Student-Newman-Keuls posthoc test was also used for between-groups comparison. In all cases, $\mathrm{p}<0.05$ was the criterion for significance. 

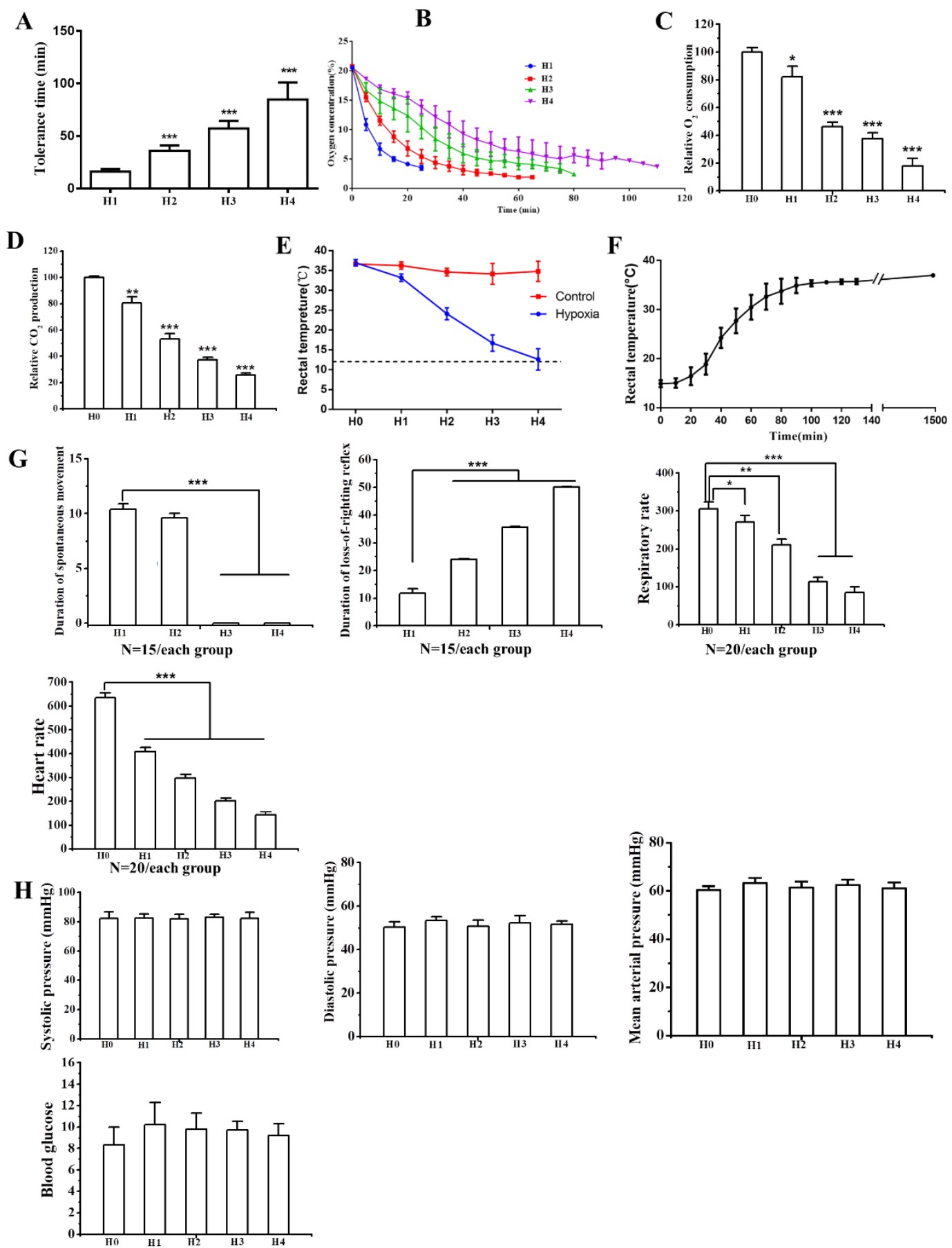

Figure 1. Hypoxia-induced hibernation reduced energy supply and demand. (A) Tolerance time in different runs of exposure to hypoxia. ***, $P<0.001 v s$. H1, N=13 each group. (B) $\mathrm{O}_{2}$ concentration in the jar. $\mathrm{N}=3$ each run. (C) Relative $\mathrm{O}_{2}$ consumption of mice exposed to hypoxia. *, $P<0.05$; ***, $P<0.001 v s$. $\mathrm{H} 0, \mathrm{~N}=12$ each group. (D) Retative $\mathrm{CO}_{2}$ production. **, $P<0.01$; ***, $P<0.001$ vs. $\mathrm{H} 0, \mathrm{~N}=12$ each group. (E) Rectal temperature at the end of each hypoxic exposure run. *, $P<0.05 ; * * *, P<0.001 v s$. H0, $\mathrm{N}=8$ each group. (F) Recovery of rectal temperature in air after exposure to hypoxia. $\mathrm{N}=5$ each group. (G) Hypoxia exposure reduced energy demand. *, $P<0.05$; **, $P<0.01$; ***, $P<0.001$. (H) Hypoxia exposure had no effect on blood pressure and blood glucose levels. $\mathrm{N}=20$ each group. 


\section{RESULTS}

\section{Hypoxia exposure reduced energy supply}

The tolerance times for when the mice were enclosed in the first jar are shown in Figure 1A; the tolerance time in each jar increased with each run. Oxygen concentration in the sealed jar was gradually reduced to about 3\% (Fig. 1B). Oxygen consumption and carbon dioxide production exponentially decreased as exposure run increased. When mice were exposed to hypoxia in the first run, their $\mathrm{O}_{2}$ consumption dropped by $\sim 43 \%$ and carbon dioxide $\left(\mathrm{CO}_{2}\right)$ output dropped by $\sim 34 \%$ (Fig. 1C). By the fourth run, $\mathrm{O}_{2}$ consumption dropped by $\sim 80 \%$ and $\mathrm{CO}_{2}$ output dropped by $68 \%$ (Fig. 1D). This drop in metabolic rate was followed by a drop in rectal temperature to $\sim 8^{\circ} \mathrm{C}$ under $\mathrm{T}_{\mathrm{a}}$ (Fig. 1E). Rectal temperature was at an average of $36.4^{\circ} \mathrm{C}$ before the first exposure and decreased to $32.9 \pm 1.3^{\circ} \mathrm{C}$ thereafter (Fig. 1E). Rectal temperature decreased gradually after each run and reached a minimum of $12.7 \pm 2.56^{\circ} \mathrm{C}$ after the fourth run, which was lower than $\mathrm{T}_{\mathrm{a}}$ (Fig. 1E). After four runs of exposure to hypoxia, the mice were returned to room air, and rectal temperature also returned to normal (Fig. 1F).
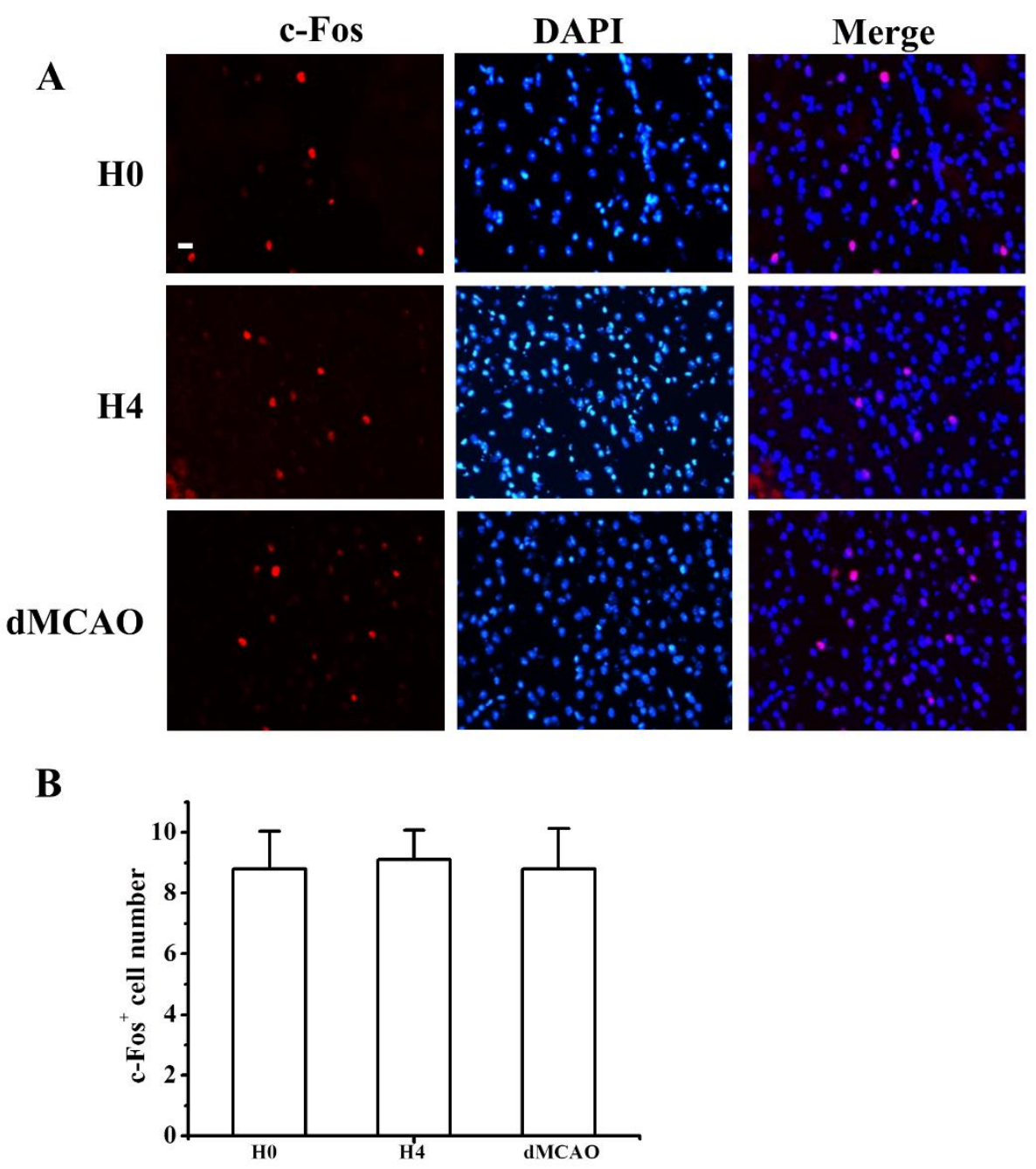

Figure 2. Comparison of c-Fos-positive cell numbers in the hypothalamus before and after hypoxiainduced hibernation. (A) Representative pictures of c-Fos-positive cells in the hypothalamic region. Scale bar $=20 \mu \mathrm{m}$. (B) Bar graph shows the quantification of c-Fos-positive cells. $\mathrm{N}=4$ each group. $\mathrm{N}=5$ each group. 


\section{Hypoxia-induced hibernation reduced energy demand}

One of the characteristics of animals in hibernation is reduced behavioral activity [23]. We first observed the spontaneous movement and righting reflex of the mice in each run. The duration of spontaneous movement of the mice was $10.4 \mathrm{~min}$ on average after hypoxia exposure at the first run (Fig. 1G). No recovery in spontaneous movement was seen after the third run. After the fourth run, the animals moved around minimally (Fig. 1G). Next, we analyzed the duration of loss-of-righting reflex (LORR) in each run $[24,25]$. The duration of LORR increased as the number of hypoxic exposure runs increased (Fig. 1G). The duration of LORR was $50.1 \mathrm{~min}$ on average during the fourth run oxygen exposure (Fig.

$1 G)$.
Heart rate and respiratory rate reduced after each run (Fig. 1G). Respiratory rate was 305 breaths per min at the beginning of the first run and decreased by $71 \%$ at the end of the fourth run (Fig. 1G). Similarly, heart beat was reduced by $75 \%$ (Fig. 1G).

\section{Hypoxia-induced hibernation had no effect on blood pressure and blood glucose}

Blood pressure remained at the same level without decreasing throughout the four runs (Fig. 1H). Blood glucose level was increased in $\mathrm{H} 1$ but remained at the same level without continuing to increase in $\mathrm{H} 2, \mathrm{H} 3$ and H4 (Fig. 1H).
A

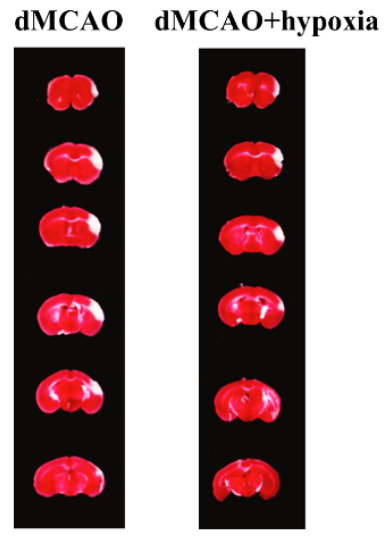

C

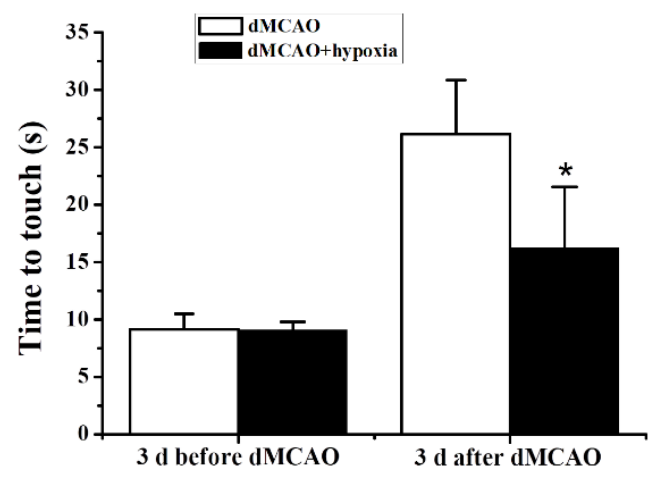

B

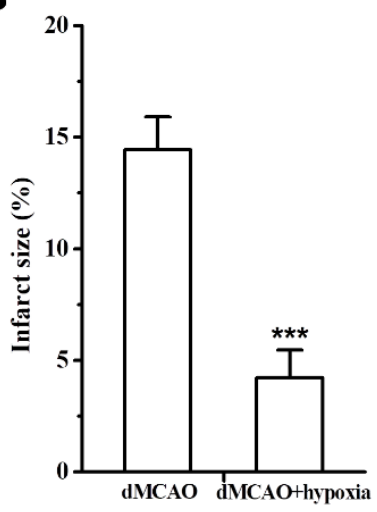

Figure 3. Hypoxia-induced hibernation reduced infarction. (A) Representative infarcts stained using 2,3,4triphenytetrazolium chloride (TTC) from the dMCAO only group and dMCAO mice treated with hypoxia. (B) Average infarct sizes. $*^{* *}, P<0.001$, vs. dMCAO only. $\mathrm{N}=7$ each group. (C) Hypoxia significantly improved sensorimotor functions after $\mathrm{dMCAO}, *, P<0.05$ vs. dMCAO only. $\mathrm{N}=12$ each group. 


\section{The effect of hypoxia-induced hibernation on neuronal activity}

It is well known that c-Fos serves as an index for neuronal activity [26]. Therefore, we investigated the number of cFos positive cells between the $\mathrm{H} 0$ and $\mathrm{H} 4$ groups in the hypothalamus as this is the thermoregulatory center in the brain with multiple nerve nuclei [27]. Our results showed that there was no significant difference between the two groups (Fig. 2), suggesting that the decline in body temperature was not a failure of body temperature regulation, but possibly an active expansion of the adaptive capacity.

\section{Hypoxia-induced hibernation reduced cerebral ischemia induced injury}

To explore whether the hypoxia-induced hibernation state had the potential for neuroprotection after large vessel occlusion, we performed focal cerebral ischemia on mice using the dMCAO method. Mice were exposed in the sealed jar for four runs at 30 minutes after dMCAO surgery. The dMCAO mice that underwent hypoxia exposure $(\mathrm{N}=7)$ averaged $4.2 \pm 1.22 \%$ infarct volumes compared with $14.4 \pm 1.49 \%$ infarct volumes in the dMCAO only $(\mathrm{N}=7)$ group (Figs. $3 \mathrm{~A}$ and $\mathrm{B})$. This $71 \%$ reduction proved to be significant $(P<0.001)$. Neurological function was evaluated using the adhesive tape removal test. Hypoxia exposure treatment significantly improved neurological performance in the adhesive tape removal test after $\mathrm{MMCAO}$ as manifested by a consistent reduction in the number of times to contact and then remove the tape from the contralateral limb (Fig 3C). One study reported that MCAO may affect hypothalamic function [28]. We therefore compared the number of c-Fos positive cells among $\mathrm{H} 1, \mathrm{H} 4$ and dMCAO mice. Our results showed that there were no significant differences among them (Fig. 2), suggesting that dMCAO did not affect hypothalamic function and that hypoxia-induced hibernation state had a direct effect on neuroprotection.

\section{DISCUSSION}

In this study, we developed a method that enabled mice to enter a hibernation-like state with the rectal temperature dropping to $\sim 8^{\circ} \mathrm{C}$ under $\mathrm{T}_{\mathrm{a}}$. The mimicked hibernation had a neuroprotective role in preventing cerebral ischemia induced injury due to occlusion of the distal MCA.

In 1960s, Dawe et al first induced hibernation state in a non-hibernator, through the injection of serum from torpid animals [11]. To date, there are several molecules that can result in reversible severe hypothermia when administered to small mammals [5]. Roth et al utilized hydrogen sulfide gas in 2005 to induce hypothermia in lab animals. The average core body temperature of these mice reached a minimum of $15^{\circ} \mathrm{C}$ in an ambient temperature of $13^{\circ} \mathrm{C}$ [29]. After receiving the glycolytic inhibitor 2deoxyglucose, hamsters readily underwent a decrease in $\mathrm{T}_{\mathrm{b}}$ below $30^{\circ} \mathrm{C}$ despite being kept in long photoperiods [30]. Mice that received 5'adenosinemonophosphate (5'AMP) were severely hypothermic, with $\mathrm{T}_{\mathrm{b}}$ as low as $25^{\circ} \mathrm{C}$ when kept in $\mathrm{T}_{\mathrm{a}}$ of about $23-24^{\circ} \mathrm{C}$ [31]. Clinically, phenothiazine drugs, chlorpromazine and promethazine can induce mild hypothermia of $32-34^{\circ} \mathrm{C}$ [7].

Hypoxia is well known to reduce the $\mathrm{T}_{\mathrm{b}}$ of mammals although the mechanism remains unclear. Tattersall et al reported that after exposure for 2 hours at $7 \%$ oxygen concentration, $\mathrm{T}_{\mathrm{b}}$ only reduced to $31.4^{\circ} \mathrm{C}$ [32]. The fall in temperature that occurs during hypoxia is the result of a reduction in the activation of thermogenic mechanisms [32]. However, the previous study has demonstrated that hypoxia can reduce body temperature to $31^{\circ} \mathrm{C}$ but could not reach the body temperature while hibernating [33]. Our study showed that repetitive hypoxia exposure can drive the mouse into severe hypothermia $12.7 \pm 2.6^{\circ} \mathrm{C}$, which is lower than an ambient temperature of $20^{\circ} \mathrm{C}$. However, our method of inducing low temperatures is different from this method of placing animals in hypobaric chambers of fixed and known concentrations. The process of our method is repetitive hypoxic exposure, and in each hypoxic exposure run, the $\mathrm{O}_{2}$ concentration in the jar is gradually reduced to about $3 \%$. In this study, the exact mechanism of hypothermia is not known. Further study into the physiological and molecular regulators driving the controlled hypoxia-induced hibernation is therefore necessary.

We then explored whether the hypoxia-induced hibernation state is neuroprotective. Our results showed that hypoxia exposure significantly reduced focal cerebral ischemia induced injury in a dMCAO model. Whether this protection via the hibernation method can be applied to other fields remains to be explored. If humans can be induced into a hibernation state, there will be significant clinical value. Hibernation therapy can have broad ranging protective effects from post ischemic infarct tissue preservation to giving transplant patients more time to await matches.

It is interesting to note that whether the hypoxia is preconditioning (before the detrimental event) or after as in our model, controlled hypoxia results in protective effects [18]. Preconditioned hypoxic animals' cerebrospinal fluids (CSF) have previously been shown to be neuroprotective for cortical rat neurons in culture, suggesting that endogenous factors exist in the CSF. The mechanism of protection between pre-hypoxic conditioning and post-hibernation states are likely similar. Altitude training and hypoxic conditioning amongst 
endurance athletes are other avenues where controlled hypoxia is utilized today. Elite swimmers training at a higher altitude have been shown to have increased hemoglobin mass, increased lactate thresholds, and faster time, suggesting a more efficient homeostasis. Erythropoietin is elevated in altitude training as well and has been found to be neuroprotective by others [34].

Post cardiac arrest patients are traditionally cooled, following the results of the hypothermia after cardiac arrest study in which both mortality and favorable neurological outcome were found to be better with mild hypothermia $32-34^{\circ} \mathrm{C}$ for 24 hours following cardiac arrest. Antidotal cases of near drowning in frigid water with neurological recovery despite prolonged circulatory arrest also support hypothermia-induced neuroprotection [35]. Furthermore, circulatory arrest and hypothermia are routinely used for surgical procedures [36]. Mild hypothermia has shown some promise in humans after acute ischemic stroke via cooling blankets/cold saline infusion [37] and endovascular selective catheter infusion of cold saline was determined to be safe [38].

While it might seem counterintuitive to place an animal undergoing a large vessel occlusion into an increasingly hypoxic environment, it is the slow controlled aspect of the hypoxia that likely results in clinical benefit. In fact, the opposite has been trialed in humans, with hyperbaric oxygen supplied to stroke patients and the authors concluded there was no benefit and could even possibly harm patients [39]. However, hyperbaric oxygen therapy in mice has been found to be neuroprotective when used as a pre-conditioning therapy [40]. Thus, many questions remain unanswered. Free divers and mountaineers are two populations of people exposed to acute and chronic hypoxia, respectively. Arterial $\mathrm{O}_{2}$ partial pressures as low as $19-23 \mathrm{mmHg}$ and $\mathrm{CO}_{2}$ levels of $16-61 \mathrm{mmHg}$ are tolerated via large increases in CBF [41]. However, this increase in CBF is likely necessary for the maintenance of consciousness and motor function, in a controlled hibernation state, such an increase in CBF would probably not be necessary. Human hibernation has recently been described as an epiphenomenon to HIV-related injury to the hypothalamus and thalamus in which the patient experiences seasonal hypothermia (nadir $31^{\circ} \mathrm{C}$ ), bradycardia, encephalopathy, and hypersomnolence made better with external warming [42]. Mild therapeutic hypothermia $\left(32^{\circ} \mathrm{C}-35^{\circ} \mathrm{C}\right)$ has been recognized as an effective neuroprotectant in experimental stroke models [43-45]. Our present study showed that severe hypothermia induced by hypoxia still has the neuroprotective role in the stroke model. Although the mechanism need to be further explored, the conceptproofed neurorpteciton would be used in a wider range of fields.
Taken together, our findings deserve a closer look with more animals to determine the best regimen for inducing hibernation. We believe that with the continuous advancement of science and technology, it will not be before long where humans can enter hibernation simply, safely, and reliably.

\section{Acknowledgments}

This work was supported by the National Key R\&D Program of China (2017YFC1308402) and Beijing Municipal Administration of Hospitals Clinical Medicine Development of Special Funding Support (ZYLX 201706).

\section{References}

[1] Geiser F (2004). Metabolic rate and body temperature reduction during hibernation and daily torpor. Annu Rev Physiol, 66: 239-274

[2] Geiser F, Brigham RM (2000). Torpor, thermal biology, and energetics in Australian long-eared bats (Nyctophilus). J Comp Physiol B, 170: 153-162

[3] Barnes BM (1989). Freeze avoidance in a mammal: body temperatures below 0 degree $\mathrm{C}$ in an Arctic hibernator. Science, 244: 1593-1595

[4] Heldmaier G, Ortmann S, Elvert R (2004). Natural hypometabolism during hibernation and daily torpor in mammals. Respir Physiol Neurobiol, 141: 317-329

[5] Lee CC (2008). Is human hibernation possible? Annu Rev Med, 59: 177-186

[6] Drew KL, Rice ME, Kuhn TB, Smith MA (2001). Neuroprotective adaptations in hibernation: therapeutic implications for ischemia-reperfusion, traumatic brain injury and neurodegenerative diseases. Free Radic Biol Med, 31: 563-573

[7] Forreider B, Pozivilko D, Kawaji Q, Geng X, Ding Y (2017). Hibernation-like neuroprotection in stroke by attenuating brain metabolic dysfunction. Prog Neurobiol, 157: $174-187$

[8] kim JY, Yenari MA (2015). Hypothermia for treatment of stroke. Brain Circul, 1: 14-25

[9] Dietrich WD, Bramlett HM (2017). Therapeutic hypothermia and targeted temperature management for traumatic brain injury: Experimental and clinical experience. Brain Circul, 3: 186-198

[10] Ratigan ED, McKay DB (2016). Exploring principles of hibernation for organ preservation. Transplant Rev (Orlando), 30: 13-19

[11] Dawe AR, Spurrier WA (1969). Hibernation induced in ground squirrels by blood transfusion. Science, 163: 298299

[12] Ramirez JM, Folkow LP, Blix AS (2007). Hypoxia tolerance in mammals and birds: from the wilderness to the clinic. Annu Rev Physiol, 69: 113-143

[13] Barros RC, Zimmer ME, Branco LG, Milsom WK (2001). Hypoxic metabolic response of the golden- 
mantled ground squirrel. J Appl Physiol (1985), 91: 603612

[14] Cross KW, Tizard JP, Trythall DA (1958). The gaseous metabolism of the newborn infant breathing $15 \%$ oxygen. Acta Paediatr, 47: 217-237

[15] Gautier H, Bonora M, Schultz SA, Remmers JE (1987). Hypoxia-induced changes in shivering and body temperature. J Appl Physiol (1985), 62: 2477-2484

[16] Wood SC (1995). Oxygen as a modulator of body temperature. Braz J Med Biol Res, 28: 1249-1256

[17] Shao G, Zhang R, Wang ZL, Gao CY, Huo X, Lu GW (2006). Hypoxic preconditioning improves spatial cognitive ability in mice. Neurosignals, 15: 314-321

[18] Lu GW, Yu S, Li RH, Cui XY, Gao CY (2005). Hypoxic preconditioning: a novel intrinsic cytoprotective strategy. Mol Neurobiol, 31: 255-271

[19] Ren C, Yao Y, Han R, Huang Q, Li H, Wang B, et al. (2018). Cerebral ischemia induces angiogenesis in the peri-infarct regions via Notch1 signaling activation. Exp Neurol, 304: 30-40

[20] Ren C, Gao M, Dornbos D, 3rd, Ding Y, Zeng X, Luo Y, et al. (2011). Remote ischemic post-conditioning reduced brain damage in experimental ischemia/reperfusion injury. Neurol Res, 33: 514-519

[21] Wang J, Ye Q, Xu J, Benedek G, Zhang H, Yang Y, et al. (2017). DRalpha1-MOG-35-55 Reduces Permanent Ischemic Brain Injury. Transl Stroke Res, 8: 284-293

[22] Liu Z, Ran Y, Huang S, Wen S, Zhang W, Liu X, et al. (2017). Curcumin Protects against Ischemic Stroke by Titrating Microglia/Macrophage Polarization. Front Aging Neurosci, 9: 233

[23] Gaglia MM, Kenyon C (2009). Stimulation of movement in a quiescent, hibernation-like form of Caenorhabditis elegans by dopamine signaling. J Neurosci, 29: 73027314

[24] Gao J, Hu Z, Shi L, Li N, Ouyang Y, Shu S, et al. (2018). HCN channels contribute to the sensitivity of intravenous anesthetics in developmental mice. Oncotarget, 9: 12907-12917

[25] Li S, Hafeez A, Noorulla F, Geng X, Shao G, Ren C, et al. (2017). Preconditioning in neuroprotection: From hypoxia to ischemia. Prog Neurobiol, 157: 79-91

[26] Tamura Y, Shintani M, Inoue H, Monden M, Shiomi H (2012). Regulatory mechanism of body temperature in the central nervous system during the maintenance phase of hibernation in Syrian hamsters: involvement of betaendorphin. Brain Res, 1448: 63-70

[27] Drew KL, Buck CL, Barnes BM, Christian SL, Rasley BT, Harris MB (2007). Central nervous system regulation of mammalian hibernation: implications for metabolic suppression and ischemia tolerance. $\mathbf{J}$ Neurochem, 102: 1713-1726

[28] Hata R, Mies G, Wiessner C, Fritze K, Hesselbarth D, Brinker G, et al. (1998). A reproducible model of middle cerebral artery occlusion in mice: hemodynamic, biochemical, and magnetic resonance imaging. J Cereb Blood Flow Metab, 18: 367-375

[29] Blackstone E, Morrison M, Roth MB (2005). H2S induces a suspended animation-like state in mice. Science, 308: 518
[30] Dark J, Miller DR, Zucker I (1994). Reduced glucose availability induces torpor in Siberian hamsters. Am J Physiol, 267: R496-501

[31] Zhang J, Kaasik K, Blackburn MR, Lee CC (2006). Constant darkness is a circadian metabolic signal in mammals. Nature, 439: 340-343

[32] Tattersall GJ, Milsom WK (2009). Hypoxia reduces the hypothalamic thermogenic threshold and thermosensitivity. J Physiol, 587: 5259-5274

[33] Geiser F (2013). Hibernation. Curr Biol, 23: R188-193

[34] Marti HH, Bernaudin M, Petit E, Bauer C (2000). Neuroprotection and Angiogenesis: Dual Role of Erythropoietin in Brain Ischemia. News Physiol Sci, 15: 225-229

[35] Huckabee HC, Craig PL, Williams JM (1996). Near drowning in frigid water: a case study of a 31-year-old woman. J Int Neuropsychol Soc, 2: 256-260

[36] Svensson LG, Crawford ES, Hess KR, Coselli JS, Raskin S, Shenaq SA, et al. (1993). Deep hypothermia with circulatory arrest. Determinants of stroke and early mortality in 656 patients. J Thorac Cardiovasc Surg, 106: 19-28; discussion 28-31

[37] Piironen K, Tiainen M, Mustanoja S, Kaukonen KM, Meretoja A, Tatlisumak T, et al. (2014). Mild hypothermia after intravenous thrombolysis in patients with acute stroke: a randomized controlled trial. Stroke, 45: 486-491

[38] Chen J, Liu L, Zhang H, Geng X, Jiao L, Li G, et al. (2016). Endovascular Hypothermia in Acute Ischemic Stroke: Pilot Study of Selective Intra-Arterial Cold Saline Infusion. Stroke, 47: 1933-1935

[39] Rusyniak DE, Kirk MA, May JD, et al. (2003). Hyperbaric oxygen therapy in acute ischemic stroke: results of the Hyperbaric Oxygen in Acute Ischemic Stroke Trial Pilot Study. Stroke, 34: 571-574

[40] Hu SL, Huang YX, Hu R, Li F, Feng H (2015). Osteopontin Mediates Hyperbaric Oxygen Preconditioning-Induced Neuroprotection Against Ischemic Stroke. Mol Neurobiol, 52: 236-243

[41] Bailey DM, Willie CK, Hoiland RL, et al. (2017). Surviving Without Oxygen: How Low Can the Human Brain Go? High Alt Med Biol, 18: 73-79

[42] Mohl L, Howell M, Buffenstein R (2018). Seasonal Hypothermia, Hypersomnolence, Encephalopathy, and Hypoventilation Following Hypothalamus Injury-A Case of Human Hibernation? Neurology, 90: 122

[43] Liu X, Wen S, Zhao S, Yan F, Zhao S, Wu D, et al. (2018). Mild Therapeutic Hypothermia Protects the Brain from Ischemia/Reperfusion Injury through Upregulation of iASPP. Aging Dis. 9: 401-411.

[44] Zhang Z, Zhang L, Ding Y, Han Z, Ji X (2018). Effects of Therapeutic Hypothermia Combined with Other Neuroprotective Strategies on Ischemic Stroke: Review of Evidence. Aging Dis. 9: 507-522.

[45] Hu H, Doll DN, Sun J, Lewis SE, Wimsatt JH, Kessler MJ, et al. (2016)Mitochondrial Impairment in Cerebrovascular Endothelial Cells is Involved in the Correlation between Body Temperature and Stroke Severity. Aging Dis. 7:14-2 